\title{
"How Far Do You Go and Where Are the Issues Surrounding That?" Dilemmas at the Boundaries of Clinical Competency in Humanitarian Health Work
}

\author{
Matthew R. Hunt, PT, PhD; ${ }^{1}$ Lisa Schwartz, $\mathrm{PhD} ;{ }^{2}$ Veronique Fraser, RN, $\mathrm{MSc}^{3}$
}

1. School of Physical and Occupational Therapy, McGill University, Montreal, Quebec, Canada; and Centre for Interdisciplinary Research in Rehabilitation, Montreal, Quebec, Canada

2. Clinical Epidemiology and Biostatistics, McMaster University, Hamilton, Ontario, Canada

3. Biomedical Ethics Unit, McGill University, Montreal, Quebec, Canada

Correspondence:

Matthew R. Hunt, PT, PhD

School of Physical and Occupational Therapy McGill University

Hosmer House 3630 prom Sir-William-Osler H3G 1Y5 Montreal, Quebec, Canada

E-mail: matthew.hunt@mcgill.ca

Conflicts of interest and funding: The authors declare no conflicts of interest. The research presented in this article was funded by a grant from the Canadian Institutes of Health Research (200703EOG). Matthew Hunt is supported by a Research Scholar Award from the Fonds de Recherche du Québec - Santé. Lisa Schwartz is supported by a private endowed chair. Veronique Fraser is supported by a stipend from the Montreal Health Equity Research Consortium (CIHR grant $\mathrm{ROH}$ 115214).

Keywords: competency; ethics; health care professional; humanitarian aid; scope of practice

\section{Abbreviation:}

NGO: nongovernmental organization

Received: September 20, 2012

Accepted: December 8, 2012

Online publication: July 26, 2013

doi:10.1017/S1049023X13008698
You go from here to there, and here you're specialized in one particular sort of thing, there you may be asked to do all sorts of things outside your specialty. How far do you go and where are the issues surrounding that?

Canadian physician discussing experiences in humanitarian aid work

Abstract

Health professionals working in humanitarian relief projects encounter a range of ethical challenges. Applying professional and ethical norms may be especially challenging in crisis settings where needs are elevated, resources scarce, and socio-political structures strained. Situations when clinicians must decide whether to provide care that is near the margins of their professional competency are a source of moral uncertainty that can give rise to moral distress. The authors suggest that responding ethically to these dilemmas requires more than familiarity with ethical codes of conduct and guidelines; it requires practical wisdom, that is, the ability to relate past experience and general guidance to a current situation in order to render a morally sound action. Two sets of questions are proposed to guide reflection and deliberation for clinicians who face competency dilemmas. The first is prospective and intended to aid clinicians in evaluating an unfolding situation. The second is retrospective and designed to support debriefing about past experiences and difficult situations. The aim of this analysis is to support clinicians in evaluating competency dilemmas and provide ethical care and services.

Hunt MR, Schwartz L, Fraser V. "How Far Do You Go and Where Are the Issues Surrounding That?" Dilemmas at the Boundaries of Clinical Competency in Humanitarian Health Work. Prehosp Disaster Med. 2013;28(5):502-508.

\section{Introduction}

Clinical and public health practice in humanitarian crises is carried out in settings where resources are scarce, needs are elevated, and social and political systems are strained or fractured. Those clinicians who travel from one country to another to provide care and assistance following a disaster or during armed conflict may also experience less clearly defined professional roles and responsibilities and decreased accountability and oversight. Health professionals who participate in international relief projects must respond to a range of ethical challenges including both familiar problems, which may be altered or amplified in the humanitarian crisis setting, as well as new issues not previously encountered in clinical work in their home country. The verbatim quotation above, along with others included in this paper, is drawn from interviews conducted during qualitative research studies with Canadian clinicians who participated in international aid work. ${ }^{1-3}$ In a study exploring ethical challenges experienced by health professionals who worked with nongovernmental organizations (NGOs) in situations of armed conflict, extreme poverty, or the aftermath of disaster, four key sources of ethical challenges were identified: (1) limited resources and the need to allocate them; (2) injustices associated with historical, political, social and commercial structures; (3) aid agency policies and agendas; and (4) norms around health professionals' roles and interactions. ${ }^{3}$ The challenges reported by research participants highlight a range of moral, and sometimes legal, questions regarding how ethics and professionalism "travel" as expatriate clinicians trained and licensed in one setting are deployed to other nations in emergency response roles. ${ }^{4}$ 
This paper focuses on a particular type of ethical challenge for humanitarian health workers: dilemmas related to deciding in situations of humanitarian action whether to provide care that is at the periphery of one's clinical training and professional competencies. For example, a physician who has assisted with, but never performed on her own, a Caesarean Section must decide whether to operate on a woman in obstructed labor because the project's surgeon is absent for several days, and the options for transferring the patient present serious concerns. ${ }^{5}$ In other cases, a nurse may consider making a decision or taking an action that would under "normal" circumstances be the responsibility of a physician, such as suturing a wound, inserting a chest tube, or setting a displaced fracture. Such dilemmas are located at the intersection of two of the themes described above: resource scarcity and norms around health professionals' roles. These dilemmas most commonly arise when human and other resources are overstretched or inadequate, and they are shaped by expectations and standards of professional and ethical practice. Such scenarios may be more common in acute relief operations during war or disaster, but they also occur in other settings of international aid work, including post-crisis reconstruction projects where resource scarcity remains pervasive, and they introduce a more nuanced set of problems than straightforward questions about malpractice. This paper begins by exploring features that contribute to shaping the moral landscape of decision-making at the boundaries of professional competency in humanitarian work. The authors describe how clinicians must apply general guidelines in particular circumstances that are often fraught with uncertainty and risk, and relate these decisions to the Aristotelian concept of phronesis (sometimes called "practical wisdom"). Two sets of questions that are consistent with phronetic practice are proposed. The first set of questions aims to support reflection and deliberation around situations in which clinicians find themselves having to choose whether or not to act at the periphery of their competency. The second set of questions aims to guide post hoc debriefing if such a decision is enacted.

\section{Dilemmas at the Boundaries of Clinical Competency in Humanitarian Health Work}

The reality of humanitarian operations, especially in the initial relief phase, is often stark: human and material resources are limited, needs are high, and few opportunities to transfer patients or consult colleagues exist. As a result, not all needs can be met and many suffer without the assistance and support they require. In humanitarian health projects, clinical care often involves prioritization and may require difficult choices of patient selection. ${ }^{6}$ Clinicians may be placed in the uncomfortable and often unfamiliar position of making allocation decisions between current patients, or between present patients and future patients. ${ }^{7,8}$ Severe resource limitations of both human and material resources are primary sources of situations when clinicians contemplate acting outside their ordinary sphere of practice or near the margins of their competency, due to a perception that better options are not available. Such a situation is described by a general surgeon from Canada with extensive experience in humanitarian aid projects, “... If a woman is in obstructed labour and there is nobody else to help, I'll do a caesarean section but you know, I prefer not to because I'm not an obstetrician and I'm not very comfortable doing it." ${ }^{9}$ This scenario is not unique and illustrates the profoundly "second-best" nature of available care in many settings of international aid and humanitarian work. In discussing these situations, it is important to distinguish between ad hoc decisions to provide care near the boundaries of clinical competence from organizational strategies of "task-shifting" that are implemented in various locales where specific tasks are shifted from higher-skilled cadres to lesser-skilled cadres or to community health workers in a regimented and deliberate approach to maximize human resources for health care delivery in humanitarian action. ${ }^{11}$

Clinicians in humanitarian work sometimes must decide whether to provide care or services beyond their core competency, training, or experience. ${ }^{5}$ Two cases presented here are drawn from the authors' empirical research and illustrate different ways in which such decisions arise and are experienced by clinicians. In certain emergency circumstances where an individual's life or bodily integrity is in jeopardy, and appropriately trained clinicians are not available, clinicians may consider providing interventions that are at the margins of or exceed the scope of practice of their disciplines or specialties. An example of this type of decision making is the following description provided by a gynecologist who was the only surgeon in an isolated region in Central Asia where a natural disaster resulted in many injuries and deaths:

I'm operating on kids, I'm operating on men, I'm thrown into a whole bunch of circumstances where I have to make a decision whether I'm going to operate or not, and knowing if I don't, the person's going to die. In many circumstances, I'm reading a book before I'm opening a belly... I have to make a decision. Which is the better of two bad options: having the wrong surgeon operate, or not operating and dying?

As in this scenario, clinicians may consider providing interventions that they would never contemplate in ordinary practice due to the degree of emergency, the lack of other feasible options, and because the consequences of not acting are judged to be dire. As described by the gynecologist, these choices are tragic ones in that they require selection of the least-worst option as each option appears morally wrong in some important way: "the better of two bad options."

In a second situation, a nurse working in a refugee camp describes the impact of choosing not to provide care that a physician would normally provide:

Sometimes I was asked to do stuff that doctors do and the child died because I didn't do it. I felt that I wasn't a doctor. I felt that I couldn't do it, but it was the only thing to do and the child ended up dying. But I didn't want the child to die because I didn't do it right either. ${ }^{12}$

As illustrated by the quotation, these choices may have dire consequences, including life-or-death decisions. Patients do or do not receive a particular type of care or intervention. As suggested by this brief narrative, decisions around providing interventions that are near, or exceed, the limits of one's competency (whether action is or is not taken) have associated moral costs. In the presentation of these types of dilemmas by research participants, the evaluation of whether to act in such circumstance is often challenging and strong feelings of discomfort and frustration were present for some in retelling an experience months or years after the event. Particularly where the stakes are highest (life or death, continued suffering or provision of relief, possibility of long-term disability), these decisions are a source of uncertainty and even angst for clinicians who must decide how to respond in a unique set of local circumstances that may even be unclear or shifting. 
A related but distinct phenomenon that also warrants attention is the possibility for acting nearer the margins of professional training or competence to become routine and integrated into the culture of humanitarian aid work, even in lessacute situations, but without being subject to sustained discussion and analysis. This possibility draws attention to the ways that particular decisions are shaped by features of the organization, as well as by contextual features of the project. Clinical competency dilemmas are also linked to other important issues in humanitarian health care, including insurance coverage, professional accountability, malpractice, resource allocation and rationing, and policy setting, as well as the important concepts of standard of care and scope of practice.

\section{Professional and Ethical Standards in Humanitarian Health Work}

In responding to ethical issues in humanitarian work, a range of ethical frameworks and approaches may be of assistance for expatriate clinicians as they address ethical issues and seek to establish their moral bearings. ${ }^{13}$ Expatriate clinicians may derive guidance from the policies and codes of conduct of the NGOs with which they are affiliated, as well as from interagency codes and charters. ${ }^{14,15}$ They may also draw on ethical codes from the professional licensing bodies in their home countries, articulations of professional and legal norms in the country where they are deployed, their professional training and prior experience, as well as international guidelines. ${ }^{16,17}$ Though informative and instructive, the guidance offered by these sources will often require interpretation. In particular, professional codes of ethics are written to be broad and encompassing, thus necessitating elucidation for how norms ought to be understood and applied in a particular setting. This breadth may leave them open for multiple interpretations, especially in settings more distant and different from the ones envisaged by the drafters of the codes.

Professional codes of practice from the home countries of expatriate clinicians were usually drafted with a different context in mind, namely a stable social and political setting and a comparatively well-resourced health system. In situations of moral uncertainty, guidance statements need to be evaluated in light of the situation at hand. Different principles or duties may need to be prioritized or reordered in response to changed circumstances, while respecting the intent of these documents as articulations of a professional morality. ${ }^{18}$

Providing care near the limits of one's competency raises the further question of appropriate professional standards of practice and whether such standards are altered or transformed in crisis settings. ${ }^{19}$ Providing assistance in an emergency situation (such as to an automobile accident victim at the side of the road) may be construed as a moral (duty of rescue) or legal (for instance, under civil law) responsibility of health professionals. Good Samaritan motivations may support clinicians to provide emergency aid to an individual in peril even if they would not routinely provide such care in their ordinary practice context. Good Samaritan laws, if present in the jurisdiction, will influence clinician liability if they act in these ways. However, humanitarian emergencies are not equivalent to car accidents. They are different in kind as well as in scale, requiring analysis of the particular clinical, social, professional, and political contexts of a crisis. Humanitarian crises also are extended in time and occur in a country with professional and legal standards that may differ from those in the home country of responders who arrive from other nations. For large-scale disasters in low-resource settings, both local and international agencies contribute to relief efforts. For expatriate clinicians working with NGOs, the policies and structures of these organizations will contribute to shaping expectations and experiences of clinical care.

The notion of "standard of care" is a critical concept from both a clinical and legal perspective. While questions of liability and legal responsibility are highly relevant to this topic and warrant careful analysis, they are beyond the scope of this paper and will not be discussed here (see IOM 2012 and Annas 2010 for more discussion of legal dimensions). ${ }^{19,20}$ Importantly, standard of care is a situation-specific evaluative standard whereby a clinician is required to act as a reasonably prudent clinician (with the same qualifications) would act in the same or similar circumstances. ${ }^{20}$ In crisis settings such as humanitarian relief operations, professional standards of care are not suspended; rather, standard of care is evaluated in light of the "facts on the ground," and in answer to the question of how a diligent professional would respond to those facts. ${ }^{19}$ But this approach does not remove the challenge of determining how a prudent clinician would act in a situation that is, by definition, emergent, complex, and critical. Determinations of what constitutes best practices may also be more difficult due to limited research on clinical care in disaster or conflict settings. In an effort to clarify best practices, the World Medical Association has urged physicians "to promote a standard competency set to ensure consistency among disaster training programs for physicians across all specialties." 21

Competency dilemmas require careful evaluation of clinical and other features that are pertinent to the situation in order to evaluate what a prudent clinician would do in the situation. Some may view patients as having a right to expect clinicians to push the boundaries of clinical competency to provide emergency assistance if there is a chance of success (whose answer to the question "which is the best of two bad options?" is that it is clearly better to have the wrong surgeon operate than not operate at all). In the home countries of expatriate clinicians, expectations for how clinicians make decisions related to scope of practice in emergency settings may be established by professional organizations. For example, in the United Kingdom the General Medical Council states that, in an emergency, physicians "must offer assistance, taking account of [their] own safety, [their] competence and the availability of other options for care." ${ }^{22}$ In the United States and Canada, national medical and nursing associations have articulated ethical duties of physicians and nurses to guide the provision of care in emergencies. ${ }^{23,24}$

In emergency situations, providing care that is beyond one's "ordinary" scope of practice may be viewed not as simply permissible or superogatory, but may be construed as a responsibility of clinicians if a better option for care does not exist and if the clinician judges that his or her competency will entail a reasonable chance of success. In other situations, clinicians who act beyond their competence, taking actions for which risks outweigh benefits, or when better care options exist, will be judged as acting wrongly.

\section{Phronesis (Practical Wisdom) and the Evaluation of Clinical Competence Dilemmas}

Decisions related to practicing near the boundaries of one's competency require careful attention. The process of deliberation and decision making can be described in relation to the 
Aristotelian notion of phronesis. ${ }^{25}$ Phronesis, often translated as practical wisdom or prudence, entails the capacity to deliberate well and to judge the correct means for achieving good ends. Individuals who employ phronesis are able to determine "how contextual particularities help to determine the forms of conduct that can be considered good under specific circumstances." 26 They will be able to identify what is morally significant in a situation and use general principles to respond in consequence.

In humanitarian work and in competency dilemmas in particular, clinicians need to be able to apply general guidance to particular circumstances. Given that decisions to act near the margin of one's competency often involve significant uncertainty, and may constitute difficult and even tragic choices (choices between two bad options), ${ }^{27}$ it is challenging to adduce the best response given the multiple features (the facts on the ground) that need to be taken into account. It is unlikely that formal guidelines will be sufficient to provide explicit guidance that clinicians always can apply, and emerge with a clear answer regarding right action. Thus, in addition to access to guidelines, clinicians need to employ phronesis to be able to evaluate a given set of circumstances, identify which features are most salient to the decision at hand, and relate general guidance and past experiences to the particular situation to make an ethically defensible decision.

\section{Questions to Support Phronetic Analysis of Competency Dilemmas}

In the following sections, two sets of questions are presented that can help support reflection and discussion around competency dilemmas and help clinicians to develop and practice phronesis. The questions emerge from the collected stories of ethical challenges from the authors' studies and reflect the tensions and questions the respondents described. These sets of questions will be most pertinent for those with less field experience and will support and focus collective decision making within teams. The aim of these questions is to support clinicians as they seek to identify morally significant factors, deliberate well, and identify an ethically defensible course of action-to support phronetic practice. The first set provides questions for reflection and deliberation in evaluating a decision that is currently being considered. These questions are aimed to structure and guide prospective decision making. The objective of the second set of questions is to support retrospective debriefing of decisions that already were made. An important aspect of phronesis is to learn from past experiences and build constructively for improving future practice. In this way, the exercise of retrospective debriefing can support clinicians to both develop and apply practical wisdom. These questions in themselves will not generate "correct" answers to the complex decisions that teams make around the limits of competency. As discussed in the previous section, phronesis is required to evaluate a morally ambiguous situation and enact ethically defensible decisions. The questions provided here are intended to stimulate reflection and wellconsidered decision making. These questions can also be used in predeparture training activities as part of case study discussions.

\section{Questions for Evaluating Situations Where Health Needs Require Care at the Margins of Individual's or Team Members' Competencies}

Circumstances can arise in which clinicians face an urgent choice whether to act at the margins of competency and, as a result, time for extended analysis or discussion is severely constrained (as in

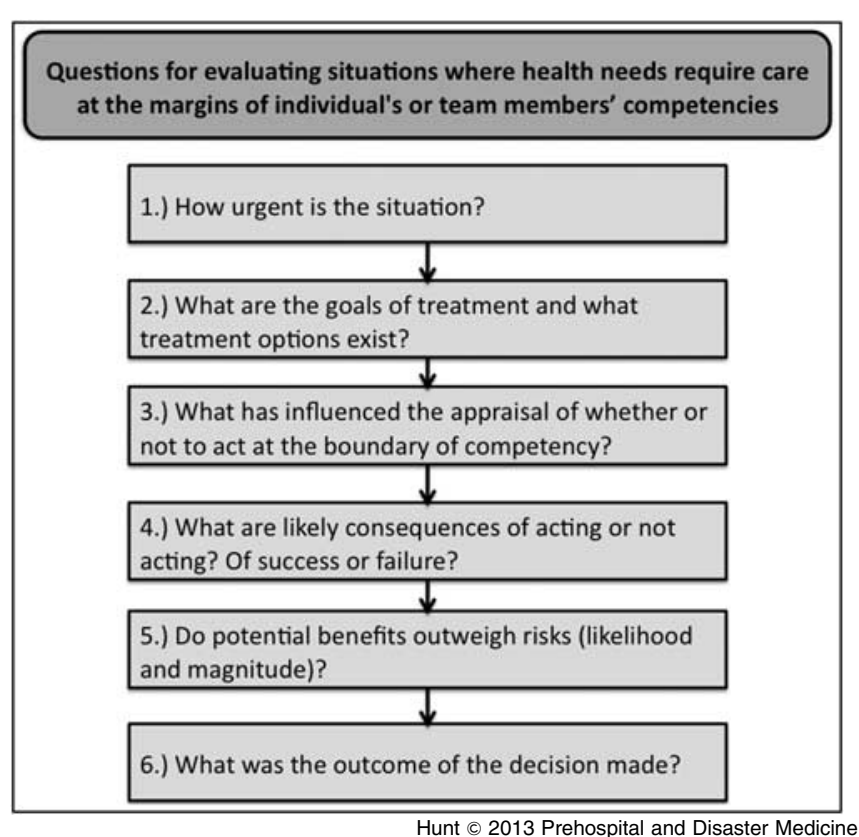

Figure 1. Questions for Evaluating a Competency Dilemma

the case of the gynecologist providing surgery on injured men in the aftermath of a natural disaster). In other cases, there is time (though limited) for discussion and reflection before action is taken (as in the case of the physician contemplating performing a Caesarean section for a woman in obstructed labor but not yet in acute distress). ${ }^{5}$ Even in the former scenario where a decision must be made quickly, deliberation is still necessary even if it is foreshortened by the pressing nature of the situation; key features still should be considered as outlined below, even if in a streamlined fashion. Suggested here and in Figure 1 are some lines of reflection and questions to pose in considering actions that are situated at the margins of individual or collective competency. These questions can help clinicians to reason well and to apply general guidance to a particular competency dilemma.

\section{How Urgent Is the Situation?}

A primary objective of humanitarian action is to save lives; this is the essence of the "humanitarian principle." ${ }^{28}$ A frequent source of competency dilemmas are situations when this goal is impeded because clinicians with the needed qualifications or training are not available or are overwhelmed by the volume and urgency of needs. A first question in analyzing such situations relates to the degree of urgency of the circumstances. Assessing urgency will enable clinicians to grasp the most salient contextual features of the scenario and thereby identify pertinent concerns and responses in a given time frame. While the primary concern may be the urgency of the patient's (or patients') health status and whether delay or transfer is possible, assessment may also involve analysis of the urgency of the broader situation. For example, clinicians might factor in to their analysis the possibility that additional casualties are expected to arrive, further taxing resources and personnel at that time. The question may then arise as to whether providing certain interventions to current patients should be deferred in order to preserve resources for others whose arrival is anticipated in the near term. This evaluation would need to be made in light of the information available; it is 
more justified if the needs of those who will arrive are better known (they are en route or will be transferred from another site).

\section{What Are the Goals of Treatment and What Treatment Options Exist?}

In considering treatment options, it is essential that the goals of care are clear. Care may be oriented toward different aims, including saving life, preserving function, or palliation. Clarity regarding treatment goals will help orient reflection on treatment options.

Before making decisions to act at the margins of their competency, clinicians should identify as many options as possible and consider a variety of alternatives. For example, the patient might be transferred to another treatment team with requisite skills and resources to provide the needed care, or a patient might benefit from another measure which falls more clearly within the competency of the clinician(s) though is generally viewed as less effective.

In some settings, humanitarian projects truly are isolated and no other options for care exist. However, there are many situations when other organizations are present and where local institutions remain functional. Health care professionals who take part in humanitarian projects should strive to be aware of what other resources are currently in place (though how much knowledge of other services can be expected will turn on the acuity and scale of the crisis, and the diversity and number of actors as well as the level of coordination among them). Transfer to other facilities is an especially pertinent option when needed interventions exceed the competency of the current treating team.

If transfer is considered, other factors might be relevant. For example, referral to another site will implicate issues related to transportation, including safety and costs, distance of the patient from family and community, and potential resource or staffing shortages in the receiving center. Such considerations ought to be accounted for and discussed with the patient, family, and treating team. In a crisis context where resources are scarce, it is also relevant to consider how a patient transferred to another team or hospital would be prioritized. It is possible that moving the patient to a central care facility would place him or her in a less favorable position on a triage list; the patient might thus not receive critical interventions at all, or the receiving unit may lack the requisite equipment or personnel to treat the patient. The overall security situation might also be factored into decisions about whether or not transferring the patient is prudent.

\section{What Has Influenced the Appraisal of Whether to Act or Not at the Boundary of Competency?}

The clinical team should take stock of both how the care or intervention needed by the patient relates to the clinical competencies of the team and what factors might influence that evaluation. For example, it might be considered how organizational and humanitarian practitioner cultures influence the response to competency dilemmas. The following description by a medical coordinator of junior physicians providing war surgery for the first time illustrates the ways in which acting beyond one's prior training may be viewed by others in humanitarian organizations:

... you have a choice, you can watch this patient die or you can say I am going to give it a try and see what happens. And it is incredible to see that some doctors have that selfconfidence to [say] OK we'll take the book out and we'll look at it-and they go for it ... And it is not all doctors who are like that but so far in my experience with (the NGO) I would say 70\% of doctors have those guts to say I have never done this but I am not going to stand here and watch this person die and not do anything.

There also may be situations when this sort of self-confidence would not be warranted, as in when acting beyond one's training and core competencies would not be what a prudent professional would do under the circumstances (it may also contravene specific professional or legal standards in the local setting). The challenge for clinicians remains to evaluate whether self-confidence or restraint is the better approach in a given situation. They first need to find out if there truly is no one else available to intervene. If transfer or referral is impossible, then proceeding with caution may be warranted. There is, however, a fine line between acting courageously and acting rashly. As such, clinicians need to practice phronetically to judge the difference. Organizational cultures and pressure from colleagues may factor in decision making and should be scrutinized.

\section{What Are Likely Consequences of Acting or Not Acting?}

Of Success or Failure?

Evaluating whether to act near the periphery of one's competency should involve an assessment of the likelihood of success; it also necessitates careful weighing of potential risks if the intervention fails (and potentially, risks associated with partial success). This evaluation will likely be challenging given that the clinicians are moving outside their core experience and training, and working in less familiar social and political contexts. The first analysis is at a clinical level, and it concerns the likelihood of clinical success and failure. Evaluation of the likely consequences for the patient of different options (eg, intervening, transferring, waiting) should be made, including considerations for short-term care, as well as follow-up and supportive care.

Evaluating professional risk is also pertinent. Before arriving at a crisis situation, clinicians and organizations should consider professional and legal liability issues, both in their home countries where they are credentialed, and in the country where they are presently working.

In some cases, it may be difficult for expatriate clinicians to identify risks, making consultation with local colleagues or national staff an important avenue to consider.

\section{Do Potential Benefits Outweigh Risks (Likelihood and Magnitude)?}

After examining questions of urgency, goals of care, influencing factors, alternate care options, and likely consequences of different options, the team needs to weigh risks and benefits across available options. Providing needed care to individuals affected by war or disaster is at the heart of the humanitarian imperative. However this imperative does not justify clinical interventions near the margins of competency when delay or referral is possible, or when risks outweigh likely benefits.

\section{What Was the Outcome of the Decision Made?}

Clinicians should also reflect on the outcome once an option has been selected and enacted. This reflection leads directly to the second set of questions listed below for post hoc debriefing. Even where a full debriefing is not planned, teams should take note of the outcome of a difficult choice and reflect on what could be learned from the situation and applied to future scenarios of 


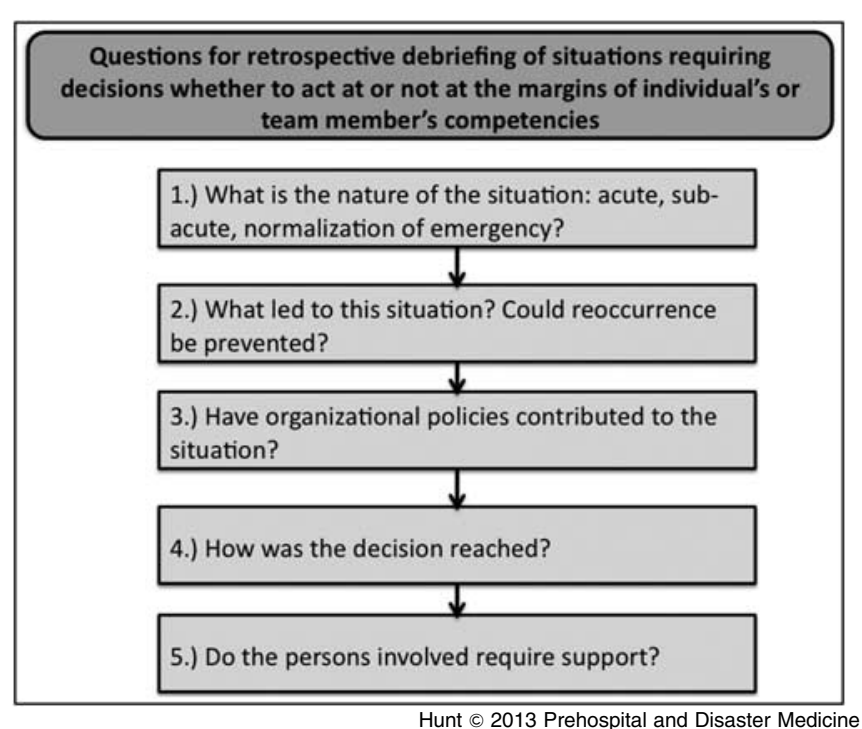

Figure 2. Questions for Retrospective Debriefing

this type. In this sense, phronesis requires more than a focus on making the best decision possible in a given situation. It requires attention to how intentions met with actual outcomes. This analysis may include assessment of outcomes for the individual patient(s) as well as consideration of impact on the broader context. However it is undertaken, follow up is essential to ensure that difficult decisions contribute to learning and improved care for future patients.

\section{Questions for Retrospective Debriefing of Situations Requiring Decisions Whether to Act or Not at the Margins of Individual or Team Competence}

Building on questions posed earlier, a further set of questions is proposed that clinicians and teams can use to analyze situations in which they were faced with choices whether to act near the periphery of their competency or not (Figure 2). These questions are particularly pertinent when a pattern exists of having to make such choices, ie, when these decisions are a recurrent feature of a project. An important component of phronesis is a commitment to learn from and integrate past experiences for comparable situations encountered in the future. These questions can support teams in this process and provide opportunities to prepare for future issues as well as to take steps to revise policy or address gaps that contributed to past situations of competency dilemmas.

\section{What Is the Nature of the Current Situation: Acute, Sub-acute, Normalization of Emergency?}

Aspects of humanitarian agency culture may support the continuation of an emergency orientation even after the acuity of the situation has decreased. ${ }^{29,30}$ An implication may be that, in certain institutional settings, clinicians are more likely to take on roles for which they have not been trained or to provide care that is outside of their usual sphere of practice. Being adaptable and willing to take on new roles are attributes important for humanitarian practitioners. Moreover, these attributes are characteristics of expertise in clinical practice. Yet cultural factors that lead to maintaining an emergency-type response in less urgent situations may also lead clinicians to act outside their core competencies, and for this to be viewed as normal and expected of them. For example, they may be less likely to delay a decision or to look at other treatment options if they maintain an emergency mentality.

\section{What Led to This Situation? Could Reoccurrence be Prevented?}

The set of features that contributed to a competency dilemma can be examined. These features may be unavoidable and the result of broader forces such as an acute increase in injuries or outbreak of disease. Others may be able to be addressed, such as lack of knowledge about available options for where patients might be transferred, including in local hospitals and clinics, and through health centers and programs of other NGOs. Examining these questions with local health care professionals or officials may improve understanding of broader implications and issues. Alternatively, there may be a need to address team and project procedures and practices, such as staffing schedules and supply issues.

\section{Have Organizational Policies Contributed to the Situation?}

Organizational features and structures can contribute to particular instances of competency dilemmas. Some examples include gaps in staffing (such as when a position on the team is unfilled or if a team member leaves for a break and is not replaced) or project mandates (such as disease-specific vertical programs in isolated settings with few other health services). Situations such as these can lead to scenarios when clinicians experience dilemmas related to competency. If clinicians identify structural features that led to a competency dilemma, they can seek to address these within the organization and thus avoid or minimize the likelihood of such dilemmas in the future, while promoting improved care for the local population. Such issues should be addressed at the appropriate levels of the organization (local team, regional team, country coordinators, headquarters).

\section{How Was the Decision Reached?}

Those participating in this retrospective evaluation should consider how a particular decision (or multiple decisions) was reached. A decision might have been taken rapidly with little or no consultation. Was this approach warranted given the circumstances and type of decision to be taken? In other circumstances, consultation among team members, or even consensus-based decision making, might have been pursued. In evaluating the situation retrospectively, it is pertinent to consider both the process of decision making and also the obstacles and barriers that were encountered. Was the decisionmaking process effective and appropriate to the situation? Should a policy or procedure be developed to assist with similar situations in the future?

\section{Do the Persons Involved Require Support?}

It is also worth considering whether any of the people involved in a particularly challenging decision-making process would benefit from additional support or debriefing. If time allows, team meetings might be organized to review the experience, or smaller meetings could be set up with those most affected by the decision. Involving outside support staff in this process, such as someone from a national office, may be beneficial in providing a different perspective on the situation. Tragic choices may result in moral residue for those making these decisions because they feel they had to choose the least-worst option, and something of moral significance had to be given up. Debriefing and collegial 
support may be valuable to support clinicians who have faced such issues and experienced feelings of angst or distress.

\section{Conclusion}

Competency dilemmas are associated with important ethical and legal issues in humanitarian work, and they are a source of ethical uncertainty for some clinicians. This paper draws on qualitative research interviews with expatriate clinicians who have experience in humanitarian work with the goal of examining different facets of this issue. The authors suggested that responding to competency dilemmas requires the exercise of phronesis (practical wisdom) in determining how best to respond. Two sets of questions were proposed to support reflection and deliberation as clinicians seek to identify the best course of action, and to support clinicians learning from difficult situations they encounter. The first set of questions is aimed at evaluating a current situation when acting near the margins of competency is considered. The second set of questions is aimed at supporting retrospective

\section{References}

1. Hunt MR. Ethics beyond borders: how health professionals experience ethics in humanitarian assistance and development work. Dev World Bioeth. 2008;8(2):56-69.

2. Hunt MR. Moral experience of Canadian health care professionals in humanitarian work. Prehosp Disaster Med. 2009;24(6):518-524.

3. Schwartz L, Sinding C, Hunt M, et al. Ethics in conditions of disaster and deprivation: learning from health workers' narratives. AJOB Prim Res. 2010;1(3): 45-54.

4. Schwartz L, Hunt M, Sinding C, et al. Western clinical health ethics: How well do the travel to humanitarian contexts?. In: Abu-Sada C, ed. Dilemmas, Challenges and Ethics of Humanitarian Action. Montreal, QC: McGill Queen's University Press; 2012.

5. Sheather J, Shah T. Ethical dilemmas in medical humanitarian practice: cases for reflection from Médecins Sans Frontières. J Med Ethics. 2011;37(3):162-165.

6. Kirsch TD, Moon MR. The Line. JAMA. 2010;303(10):921-922.

7. Fox RC, Goemaere E. They call it "patient selection" in Khayelitcha: the experience of Médecins Sans Frontières-South Africa in enrolling patients to receive antiretroviral treatment for HIV/AIDS. Camb Q Healthc Ethics. 2006;15(3):302-312.

8. Sinding C, Schwartz L, Hunt M, et al. "Playing god because you have to": Canadian health professionals' experiences of rationing care in humanitarian and development work. Public Health Ethics. 2010;3(2):147-156.

9. Schwartz L, Sinding C, Hunt M, et al. Ethics in conditions of disaster and deprivation: learning from health workers' narratives. AJOB Prim Res. 2010;1(3):51.

10. Terry F. Condemned to Repeat?: The Paradox of Humanitarian Action. Ithaca, NY: Cornell University Press; 2002:216.

11. Janneck L, Cooper N, Frehywot S, Mowafi H, Hein K. Human Resources in Humanitarian Health Working Group Report. Prehosp Disaster Med. 2009; 24(Suppl 2):s184-s193.

12. Hunt MR. Ethics beyond borders: how health professionals experience ethics in humanitarian assistance and development work. Dev World Bioeth. 2008;8(2):65.

13. Hunt M. Establishing moral bearings: ethics and expatriate health care professionals in humanitarian work. Disasters. 2011;35(3):606-622.

14. The Sphere Project: Humanitarian Charter and Minimum Standards in Disaster Response. The Sphere Project 2011. http://www.sphereproject.org/resources/downloadpublications/ ?search $=1 \&$ keywords $=$ \&language $=$ English\&category $=22$. Accessed May 10, 2012.

15. The International Federation of Red Cross and Red Crescent Societies and the ICRC: The Code of Conduct for the International Red Cross and Red Crescent Movement and Non-Governmental Organizations (NGOs) in Disaster Relief. debriefing following a situation (or recurrent situations) when a decision whether or not to act at the periphery of competency was made. Discussion of these questions will not necessarily lead to resolution of the issue, avoidance of malpractice legal actions, or removal of moral distress for those involved, but the goal of this process is to support a more comprehensive analysis of the situation so that the team can enact well-considered and ethically defensible actions.

\section{Acknowledgements}

The authors thank the respondents of the research studies discussed in this paper, as well as the members of their broader research team (Naomi Adelson, Sonya de Laat, Jennifer Ranford, Christina Sinding, Laurie Elit, and Lynda Redwood-Campbell) for their contributions to their thinking around these issues. They also thank participants in the Global Health Ethics Works-inProgress group at McGill University for their helpful comments on an earlier draft of this paper.

http://www.ifrc.org/en/publications-and-reports/code-of-conduct/. Accessed May 10, 2012.

16. World Medical Association. WMA International Code of Medical Ethics. http:// www.wma.net/en/30publications/10policies/c8/. Accessed May 10, 2012.

17. International Council of Nurses. The ICN Code of Ethics for Nurses. http:// www.icn.ch/about-icn/code-of-ethics-for-nurses/code-of-ethics-for-nurses-629.html. Accessed May 10, 2012.

18. Schwartz L, Hunt M, Sinding C, et al. Models for humanitarian health care ethics. Public Health Ethics. 2012;5(1):81-90.

19. Annas GJ. Standard of care: In sickness and in health and in emergencies. N Engl J Med. 2010;362(22):2126-2131.

20. Institute of Medicine. Crisis Standards of Care: A Systems Framework for Catastrophic Disaster Response. http://www.nap.edu/catalog.php?record_id=13351. Accessed May 10, 2012.

21. World Medical Association. WMA Declaration of Montevideo on Disaster Preparedness and Medical Response. http://www.wma.net/en/30publications/10policies/d3/ Accessed May 10, 2012.

22. General Medical Council. Good Medical Practice. www.gmc-uk.org/guidance/ good_medical_practice.asp. Accessed May 10, 2012.

23. Canadian Nursing Association. Code of Ethics for Registered Nurses. http://www2.cnaaiic.ca/cna/documents/pdf/publications/Code_of_Ethics_2008_e.pdf. Published 2008:46. Accessed May 10, 2012.

24. Canadian Medical Association. Code of Ethics. http://policybase.cma.ca/dbtw-wpd/ PolicyPDF/PD04-06.pdf. Published 2004: section 18. Accessed May 10, 2012.

25. Aristotle. The Nicomachean Ethics. Ross D-trans. Ackrill JL, Urmson JO-revis. Oxford, UK: Oxford University Press; 1980.

26. Carnevale FA. The birth of tragedy in pediatrics: a phronetic conception of bioethics. Nurs Ethics. 2007;14(5):576.

27. Nussbaum MC. The Fragility of Goodness: Luck and Ethics in Greek Tragedy and Philosophy. New York, NY: Cambridge University Press; 2001.

28. Pictet J. Development and Principles of International Humanitarian Law. Dordrecht, The Netherlands: Martinus Nijhoff Publishers; 1985.

29. Hilhorst D, Schmiemann N. Humanitarian principles and organisational culture: everyday practice in Médecins Sans Frontières Holland. Dev in Practice. 2002;12(3-4): 490-500.

30. Hunt MR. Resources and constraints for addressing ethical issues in medical humanitarian work: experiences of expatriate healthcare professionals. Am J Disaster Med. 2009;4(5):261-271. 